\title{
Sven Schürkes*
}

\section{Das kyōgen-Stück Rōmusha (Die alten Krieger)}

https://doi.org/10.1515/asia-2019-0010

\section{The warrior kyōgen Rōmusha}

\begin{abstract}
This paper presents a translation of the kyōgen play Rōmusha based on the text from the Toraakibon of the Ōkura (mid seventeenth century) school. It features the role of a boy acolyte within the homoerotic tradition of Buddhist temples in premodern Japan. Those boys were usually termed chigo. While there is already a considerable amount of research done on the historic circumstances and literary conventions in other genres, their appearance in kyōgen theater may add fruitful insights and also shed some light on their function in the comical arts. In the recent years Rōmusha has been performed again several times and it offers a vivid, realistic and erotic atmosphere which is rare to be seen in classical kyōgen. The paper aims to illustrate the structure of the play. While the focus is on the role of the chigo, comical aspects of the drama, references to $n \bar{o}$ theatre and different interpretations and performance practices will also be mentioned.
\end{abstract}

Schlagworte: Homosexualität, Lustknabe, Tempelknabe, Homoerotik, alte Krieger

Keywords: Homosexuality, boy acolyte, page, Homoerotic, old warriors

\section{Das Greisenstück Rōmusha: Zwischen Knaben- und Jünglingsliebe?}

Rōmusha 老武者 (Die Alten Krieger) ist in vielerlei Hinsicht ein außergewöhnliches Stück außerhalb des klassischen Repertoires im kyōgen 狂言. Es treten zwei Gruppen von Darstellern auf, was im kyōgen als tachishu 立衆 bezeichnet wird und für eine seltene Geschäftigkeit auf der Bühne sorgt. Der aufschlussreichste Aspekt ist allerdings, dass die Rolle eines chigo 稚児 zentrales Element des Plots

*Corresponding author: Sven Schürkes, FB II Japanologie, Universität Trier, Trier, Germany. E-mail: schuerkes@uni-trier.de 
ist. ${ }^{1}$ Bei dem Terminus chigo handelt es sich in diesem Kontext um eine Bezeichnung für religiös-erotisch kontextuierte Tempelknaben aus dem japanischen Mittelalter und der Neuzeit, welche bereits in der Prosa und der stärker lyrischen Theatergattung $n \bar{o}$ 能 Grundlage für die Entstehung einer Reihe von Erzählungen und Dramen mit eigens ausgeprägtem Topos sind. Die Vorstellung dieser heiligen Knaben hatte einen deutlichen Einfluss auf die bildenden Künste vor allem im Mittelalter, zum Beispiel im Bereich von erotischen Holzschnitten (shunga 春 (画) oder der buddhistischen Ikonographie. Rōmusha ist eines der wenigen Stücke im kyōgen, welches die Transformation zum geziemteren Hoftheater beim Übergang in das 17. Jahrhundert überstanden hat und heute noch erahnen lassen kann, wie frivol diese Theaterform zu sein vermochte. Es ist zudem ein Zeugnis davon, in welcher Weise chigo in den humorvolleren Aufführungskünsten ausgestaltet worden sind, denn mit den buddhistischen Heilsbringern aus den ernsteren Gattungen hat der chigo in Rōmusha kaum noch etwas gemein. Bisher gibt es noch keine Übersetzung in eine westliche Sprache, deshalb soll das Stück an dieser Stelle vorgestellt und untersucht werden. Ausgangstext für die Übersetzung ist die Version aus dem Toraakibon ${ }^{2}$ der Ōkura-Schule. ${ }^{3}$

Die Hauptrolle (shite シテ) in Rōmusha ist ein Greis aus der Umgebung, in Nebenrollen (ado アド) treten jeweils ein Gastwirt, ein chigo, ein sanmi 三位, eine Gruppe von Burschen sowie eine Gruppe von Greisen auf. Der Begriff sanmi, oder in der alternativen Lesung auch san’i, bezeichnet im weitesten Sinne einen Hofbeamten des dritten Ranges. Im buddhistischen Kontext referiert er auf Priester von niedriger Stellung, insbesondere in ihrer Funktion als Vormund eines chigo. ${ }^{4}$ Dementsprechend handelt es sich in diesem Fall um einen Priester

1 Weitere kyōgen-Stücke mit einem chigo als eine der Dramatis Personae: Kanazu jizō (Die Jizō-Statue von Kanazu), Chigo yabusame (Das Bogenschussritual); Weitere kyōgen-Stücke mit eindeutigem chigo-Bezug: Fumi-ninai (Die Last der Liebe), Uguisu (Der Japanbuschsänger); bei dem Stück Kumo der Ōkura-Schule, das nur fragmentarisch erhalten ist, ist der Bezug interpretierungsbedürftig. Hashimoto argumentiert hier für einen chigo-Bezug. Siehe Hashimoto 2005: 137.

2 Das Kyōgen no hon von Ōkura Toraakira (1597-1662), besser bekannt unter dem Namen Toraakibon, ist eine der ersten Textsammlungen von kyōgen-Stücken aus dem Jahre 1642. Neben der Ōkura-Schule ist heute nur noch die Izumi-Schule aktiv. Sagi ist die letzte der drei großen Schulen, von ihr sind nur Dramentexte und Aufzeichnungen überliefert.

3 Siehe Ikeda/Kitahara 1972: 136-140.

4 Siehe Kitahara 2000: Bd. 8: 403. Auch Hashimoto versteht sanmi als priesterlichen Vormund eines chigo. Siehe Hashimoto 2005: 134. Im Nōgaku daijiten heißt es hingegen, dass die Bedeutung von sanmi unklar sei. Es wird darauf hingewiesen, dass in dem nō-Stück miidera ebenfalls ein Priester als sanmi-dono 三位殿 bezeichnet wird und es Erklärungsversuche gibt, wonach es sich dabei um eine abgewandelte Form des Begriffs shami 沙弥 handeln könnte. Dies ist wiederum eine Bezeichnung für Personen, die sich erst frisch dem Buddhismus verschrieben haben. Siehe Kobayashi et al. 2012: 954. 
und einen Lustknaben. $\mathrm{Zu}$ beachten ist, dass die Jünglinge in diesem Stück mit dem Ausdruck wakashu 若衆 bezeichnet werden. Hiermit sind jedoch nicht die homoerotisch konnotierten Samurai-Knappen oder Prostituierte aus dem Kabuki gemeint, sondern es handelt sich im wörtlichen Sinne um junge Burschen aus der Gegend. ${ }^{5}$ Das Grundgerüst des Stücks ist rasch zusammengefasst: Ein sanmi begleitet einen chigo nach Kamakura, um dort mit ihm Sehenswürdigkeiten zu besichtigen. Die beiden reisen inkognito und machen einen Zwischenstopp in einer Herberge. Einige Burschen aus der Umgebung hören von der Ankunft des reizenden Knaben und wollen mit ihm Reiswein trinken. Nach einiger Taktiererei gelingt es ihnen, durch die Vermittlung des Gastwirts den sanmi zur Einwilligung $\mathrm{zu}$ überreden, und sie veranstalten ein Trinkgelage. Ein Greis aus der Umgebung hört ebenfalls von dem Knaben und entschließt sich, den Gastwirt auch um ein Treffen mit dem chigo zu bitten. Er wird jedoch mit Verweis auf die Burschen abgewimmelt und kommt daraufhin erzürnt mit einer Meute weiterer Greise wieder, um das Gasthaus zu stürmen. Nach einem wilden Kampf müssen die Burschen ihre Niederlage eingestehen und die Greise tragen den chigo küssend von der Bühne. ${ }^{6}$

Es existieren verschiedene Versionen in Stücksammlungen aller drei Schulen. Ein Großteil der textlichen Unterschiede ist zwar marginal, das Ende unterscheidet sich allerdings erheblich und bietet Anlass zur Interpretation. In dem Text aus dem Toraakibon lässt es sich so verstehen, dass die Burschen den Kampf verlieren und die Greise den chigo küssend von der Bühne tragen. Somit ist das Stück besonders für die Ōkura-Schule ungewöhnlich explizit und erotisch. In der heutigen Aufführungspraxis wird das Ende laut Hashimoto hingegen anders verstanden und dementsprechend aufgeführt. Die Zeile „omoi no hoka naru nyakuzokuzuki shi“ (über alle Erwartung lieben sie Jünglinge) deutet man als überraschenden Wendepunkt, an dem die Greise durch den eng umschlungenen Kampf von den Burschen so angetan sind, dass ihr erotisches Interesse von dem Knaben auf die wakashu umschlägt. Der Kampf um den chigo findet damit ein Ende, und alle gehen mit ihm voran zufrieden von der Bühne ab. ${ }^{7}$ Die Schlussszene bedarf also einer etwas genaueren Betrachtung.

Die Ursache für die divergierenden Interpretationen mag im Begriff nyakuzoku 若族 liegen. Das Kokugo daijiten definiert ihn als Ausdruck für Jugendlicher (wakai mono 若い者) oder im engeren Sinne auch als Ausdruck für Jugendlicher mit homoerotischem Bezug. ${ }^{8}$ Somit kann nyakuzoku auf die gleiche Gruppe von

\footnotetext{
5 Siehe Dōmoto 1976: 22.

6 Für eine Zusammenfassung siehe Koyama 1990: 472.

7 Siehe Hashimoto 2005: 134-135.

8 Siehe Kitahara 2000: Bd. 10: 508.
} 
Personen referieren wie wakashu in seiner unspezifischen Bedeutung. Zudem hat wakashu mit der Bezeichnung chigo zur Entstehungszeit des Stückes eine Phase semiotischer Überlappung durchlaufen. Der Begriff erlaubt daher ohne kontextuelle Einordnung keinen deutlichen Rückschluss auf das Alter einer männlichen Person. ${ }^{9}$ Beide Begrifflichkeiten lassen also im zeitgenössischen Gebrauch eine Trennschärfe vermissen, die aus heutiger Sicht zunächst evident erscheint. Nyakuzoku bezieht sich folglich nicht notwendigerweise auf jugendliche Burschen, sondern kann ebenso jüngere Knaben umfassen. Selbst wenn man dieser Annahme nicht folgen möchte, muss man zudem berücksichtigen, dass der Begriff chigo im buddhistischen Kontext kein definitiv präpubertäres Alter voraussetzt. Chigo in Tempeln waren realiter nicht nur jüngere Knaben, sondern gleichfalls ältere Jugendliche oder gar junge Erwachsene. ${ }^{10}$ Es lässt sich nicht ausschließen, dass der chigo in Rōmusha eigentlich ein Jugendlicher ist oder nyakuzoku-zuki 若族好き lediglich ein allgemeines homoerotisches Interesse bezeichnet. Man kann diese Zeile demnach entweder so interpretieren, dass die Greise über alle Erwartung den chigo lieben und daher so heftig kämpfen, dass ein Sieg für die Burschen aussichtslos ist. Oder man interpretiert es entsprechend der heutigen Aufführungspraxis so, dass nyakuzoku exklusiv die etwas älteren Burschen meint, die Greise also nun durch die Rauferei auf die wakashu fixiert sind und darüber den Knaben vergessen. ${ }^{11}$ Das Tenribon ${ }^{12}$ der Izumi-Schule weist in diese Richtung, da dort bei dem zweimal gesungenen Vers in Lautschrift einmal nyakuzuku-zuki und einmal konkret wakashu-zuki angegeben wird. ${ }^{13}$ Die Regieanweisung im Toraakibon hebt jedoch die Ambiguität des Begriffs nyakuzoku in Richtung des Knaben auf. Dort steht, dass der chigo küssend von der Bühne getragen wird, während der Wirt, der sanmi und die Burschen nach Hause gehen. Es wird zwar nicht expliziert, wer den chigo von der Bühne trägt, aber da der Hauswirt und der sanmi im gesamten Stück keinerlei erotisches Interesse an dem Knaben gezeigt haben, bliebe sonst niemand, der ihn sonst küssend von der Bühne tragen könnte. Man kann zwar davon ausgehen, dass ein priesterlicher Vormund eine erotische Beziehung zu

9 Für eine Diskussion der semiotischen Überlappung von chigo und wakashu siehe Iwata 2002: 136-140.

10 Es gibt bislang keine umfassende Untersuchung des Alters der chigo, dies kann hier aus Platzgründen auch nicht geschehen. Als Nachweis für das Argument mag eine kurze Einschätzung auf Grundlage literarischer Quellen von Paul Atkins dienen, wonach chigo bis zu 19 oder in Einzelfällen sogar 26 Jahre alt sein konnten. Siehe Atkins 2008: 949.

11 Siehe Hashimoto 2005: 135.

12 Das Kyōgen rikugi, meist als Tenribon bezeichnet, ist eine Textsammlung der Izumi-Schule und aus dem Jahre 1646, es ist also wenige Jahre nach dem Toraakibon entstanden.

13 Siehe Kitagawa 1994: 349. 
seinem Knaben haben mag, aber die Struktur des Stückes legt dies nicht nahe. Der Fokus des erotischen Interesses liegt eindeutig bei den Burschen und den Greisen. In dieser Version erobern also die Greise den Knaben.

Hashimoto hebt hervor, dass in Rōmusha die Parodie von Kampfstücken aus dem Nō einen Teil der Komik ausmacht. ${ }^{14}$ So könnte laut Dōmoto die weiter unten übersetzte Zeile ,ikani ikioi tamō tomo, sashitaru koto wa, arajimono wo to, ichido dottozo waraikeru“ aus Rōmusha eine Abwandlung aus dem heute nicht mehr aufgeführten Stück Moriya 守屋 sein. Der Mittelteil der von Dōmoto zitierten Stelle aus diesem nō-Stück weist zwar semiotisch kaum Gemeinsamkeiten auf, die syntaktische Struktur ist aber auffallend ähnlich. So heißt es dort „ikani [...] tomo, [...], ichido dottozo waraikeru“. ${ }^{15}$ In Moriya wird der Prinz Shōtoku Taishi von Mononobe no Moriya gejagt, bis er von Holzfäller-Greisen gerettet wird. Als eine übermächtige Streitmacht von Moriya dazustößt, verschwindet Shōtoku Taishi in dem Getümmel. Später erhält er durch seine regelmäßigen Gebete Hilfe von der Shinto-Gottheit Kasuga Myōjin und vernichtet Moriya und dessen Armee. ${ }^{16}$ Hier stellen sich also ebenfalls Greise im Kampf gegen eine Schar von wesentlich jüngeren Kriegern und beide Seiten streben nach dem Prinzen Shōtoku Taishi. In diesem Fall wollen die Greise ihn allerdings lediglich beschützen, während die jungen Krieger die Absicht haben, ihn zu erschlagen eine sexuelle Komponente ist hier nicht denkbar. Es gibt jedoch eine ganze Ikonographie von Shōtoku Taishi als reizvollen und göttlichen chigo, ${ }^{17}$ daher scheint es zumindest möglich, dass dies wenigstens inhaltlich als Inspiration für Rōmusha gedient haben könnte. Auch die Aufzählung von legendären Greisenkriegern am Ende des Stücks setzt deutliche nō-Referenzen. So verweist die Erwähnung von Sanemori Saitō offensichtlich auf das Stück Sanemori, in welchem seine Heldentaten auf dem Schlachtfeld im hohen Alter besungen werden. ${ }^{18}$ Der starke Kontrast zwischen den lyrischen Beschreibungen des alten Kriegers in Sanemori - nobel und anmutig - und den lüsternen Greisen mit ihren rostigen Waffen in Rōmusha steigert die komische Wirkung an dieser Stelle. Auch die Erwähnung von Kumasaka referiert auf das gleichnamige $N \bar{o}$ Stück, in dem eindrucksvoll der Kampf zwischen dem Banditen und dem 16jährigen Ushiwakamaru - dies ist der Jugendname des legendären Feldherren Minamoto no Yoshitsune - lyrisch verarbeitet wird. ${ }^{19}$ In diesem Fall hat der alte

14 Siehe Hashimoto 2005: 135.

15 Siehe Dōmoto 1998: 711-712; Siehe auch Hashimoto 2005: 136.

16 Siehe Kitahara 2000: Bd. 2: 1406.

17 Siehe hierzu Guth 1987: 9-13.

18 Siehe Koyama 1989: 110-112.

19 Siehe Koyama 1989: 456-458. 
Krieger keine Chance gegen die Kampfkunst des jungen Burschen und stirbt schließlich. So erzeugen beide Anspielungen die Erwartung, dass die Greise kaum siegreich aus dem Kampf hervorgehen können, und lassen deren Drohung für das wissende Publikum lächerlich wirken.

Die zentrale Bedeutung des chigo als Kulminationspunkt der Handlung steht im Kontrast zu seiner Handlung auf der Bühne. Obwohl er im Gegensatz zu anderen chigo-Stücken tatsächlich auftritt, bleibt er über weite Strecken passiv. Improvisatorische Dialogteile sind nicht mehr historisch rekonstruierbar, aber im Dramentext ist keine Zeile für ihn festgehalten und er nimmt auch keinen aktiven Einfluss auf das Geschehen. Es wird gleich zu Beginn aber deutlich, dass er von hohem Range sein muss, da der sanmi von ihm als saru okata さる御方 spricht. ${ }^{20}$ Außerdem erklärt der sanmi, dass er ihn deshalb nach Kamakura begleitet, weil dies der Wunsch des Knaben ist. Da er diesem Wunsch nicht primär aus Zuneigung nachkommt - so etwa der Fall in dem kyōgen-Stück Uguisu - handelt es sich um ein Geheiß des Knaben an den sanmi. Erst bei dem Trinkgelage rückt der chigo stärker in den Vordergrund: dort verlangt er nach süßen Klößen, singt und tanzt zusammen mit dem sanmi närrisch umher (ahōge ni mau). Laut Hashimoto ist dies der Punkt, wo das Stück beginnt, zum kyōgen zu werden. ${ }^{21}$ Obwohl der Knabe von hohem Range ist und als chigo eine strenge Etikette befolgen musste, ${ }^{22}$ verschlingt er gierig Süßigkeiten und tänzelt in lächerlicher Weise. Dem Publikum waren die eleganten und religiös überhöhten Knaben bestens aus den Erzählungen und nō-Stücken bekannt. ${ }^{23}$ Dieser Topos wird in Rōmusha aufgegriffen und durch den scharfen Kontrast zwischen dem buddhistischem Ideal und der hier gezeigten Realität entzaubert; die vermeintliche Heiligkeit des Knaben wird ins Komische verschoben. ${ }^{24}$ In der Izumi-Version aus dem Tenribon heißt es an entsprechender Stelle zwar lediglich shōjin mo mau (der Knabe tanzt ebenfalls) und die Regieanweisung bleibt vage. Eine Konkretisierung war allerdings auch nicht notwendig, da die hier erforderliche Art und Weise des Tanzes entsprechend bekannt war. In der Textsammlung Namigatabon der Izumi-Schule aus der Mitte der Edozeit (1600-1868) heißt es etwas konkreter, dass in deutlich ausgelassener Fasson im Takt getanzt und mit den Händen geklatscht wird. ${ }^{25}$

20 Dies ist unter anderem eine ehrerbietige Bezeichnung für adlige Kinder. Siehe Kitahara 2000: Bd. 12: 1018.

21 Siehe Hashimoto 2005: 136.

22 Für eine Darstellung der überaus strikten Verhaltensregeln für chigo aus dem shukaku hosshinnō 守覚法親王 siehe Hiramatsu 2007: 100-104.

23 Für eine Diskussion des Topos in den chigo monogatari siehe Childs 1980 oder Atkins 2008, für eine überblicksartige Darstellung desselben im Nō-Theater siehe MacDuff 1996.

24 Siehe Dōmoto 1976: 34.

25 Siehe Hashimoto 2005: 135. 
Für ein Publikum, das den chigo-Topos der ernsten Gattungen oder die tatsächlichen Sitten um die Tempelknaben kannte, muss gerade diese Parodie äußerst humorvoll gewesen sein. Das offene Ende, welches Raum für die Vorstellung der eventuell folgenden erotischen Handlungen gelassen hat, trug vermutlich ebenso zur Popularität von Rōmusha bei. Dōmoto vermutet ferner, dass das Stück durch den Kampf und das große Getümmel auf der Bühne auch einen zutiefst realistischen Eindruck vermittelt hat und der starke Kontrast des Kampfs zwischen altersschwachen Greisen und jugendlichen Burschen eine bis heute nachvollziehbare komische Komponente ausmacht. ${ }^{26}$ Dazu trägt auch die ōjiMaske des Greisen bei, die ein heiter-humorvolles Gesicht darstellt, das allerdings eine schlaffe Haut offenbart und voller runzliger Falten ist. Einige Ausgestaltungen der öji-Maske zeigen einen halb geöffneten Mund mit Zahnlücken und vereinzelten Zähnen, was wunderbar zu der Charakterisierung aus dem issei passt. $^{27}$ Das issei - eine kurze Ballade - ist folglich die Stelle, an welcher der Gegensatz zwischen Jung und Alt kulminiert. In geschickter Weise wird hier die Gebrechlichkeit der Greise durch das Kopfwissenwort „azusa no yumi“ (= Bogen aus azusa-Holz; evoziert eine Vorstellung von krumm oder knorrig) bildlich vor Augen geführt, auch die homonym verschachtelte Beschreibung „okina sabitaru yarinaginata“ (okina sabi= wie alte Männer; sabitaru yarinaginata = verrostete Waffen) trägt zu dieser Wirkung bei, indem es die Alten als eingerostete Tattergreise illustriert. Nachdem die Burschen dann mit ihrer jugendlichen Stärke prahlen, fällt den Alten nichts Besseres ein, als Großtaten berühmter Greise aufzuzählen und damit zu drohen, dass sie durch ein langes Leben abgehärtet sind und selbst mit 70 oder 80 Jahren die Burschen noch schlagen können. Dass sie dann nach dieser kaum ernstzunehmenden Drohung tatsächlich siegreich sind, krönt das Ende des Stücks.

Im Toraakibon zählt Rōmusha zu den Erststücken, welche innerhalb des kyōgen einen segensverheißenden Zweck erfüllen. Scholz-Cionca erklärt die Verschiebung des Stückes an diese Stelle mit einer Frauenphobie Ōkuras, der nicht nur etliche erotische Szenen aus anderen Stücken gekürzt, sondern auch sämtliche Frauenstücke aus den Erstspielen verbannt hat. An die Stelle des alten Lüstlings aus dem Stück Makura monogurui tritt bei Ōkura laut Scholz-Cionca hier der Greis aus Rōmusha, das Stück verfolgt gleichsam eine lebensverlängernde Wirkung. Da hier der chigo allerdings fern von den idealisierten Knaben der ernsteren Gattungen dargestellt und dieser Topos sogar bewusst persifliert wird, sieht sie Rōmusha eher in der Tradition edozeitlicher homophiler Kurzge-

26 Siehe Dōmoto 1976: 26-27.

27 Für eine Beschreibung und Abbildung der Maske siehe etwa Kobayashi 2004: 63. 
schichten wie Kinō wa kyō no monogatari. ${ }^{28}$ Offenbar steht dabei aber auch die bereits beschriebene komische Offenlegung buddhistischer Knaben-Apotheose im Vordergrund, denn auch Stücke wie Kanazu jizō oder Chigo yabusame greifen dies auf: Im erstgenannten Stück gibt sich ein schelmischer Knabe als Bodhisattva Jizō aus, nur um wie in Rōmusha nach Klößen und Reiswein zu gieren und ganz profan zu feiern. Im zweitgenannten Stück verkleidet sich eine Betrügerin zunächst erfolgreich als chigo und entweiht dabei ein heiliges Bogenschuss-Ritual, bis der Schwindel schließlich auffliegt.

Sowohl die eingangs besprochene Frage nach dem Alter des chigo, als auch die Entscheidung für oder gegen die Fokussierung auf eine segensspendende Funktion des Stückes durch Erotik sind maßgeblich für Rekonstruierungsversuche. Die Ōkura-Schule hat sich in der Aufführung von 1992 mit Yamamoto Norihide (geb. 1979) für einen vergleichsweise älteren Darsteller entschieden, sie bleibt nah am überlieferten Skript mitsamt erotischen Anspielungen und hebt die feierliche, lebensverlängernde Stimmung des Stückes hervor. Die IzumiSchule hingegen wählte in der Aufführung von 2005 mit Nomura Yuuki (geb. 1999) einen sehr jungen Kinderdarsteller. Die progressivere Aufführung enthält mehr Elemente, die nicht tradiert sind, rückt das Verhältnis zwischen sanmi und chigo in die Nähe einer humorvollen Vater-Sohn-Beziehung und strebt insgesamt eine heitere Stimmung an. Das Ergebnis sind zwei grundverschiedene und überaus interessante Rekonstruierungsversuche desselben Stücks. ${ }^{29}$

\section{Die Alten Krieger}

Sanmi (shidai ${ }^{30}$ ): Wenn du heimlich auf Reisen bist, wenn du heimlich auf Reisen bist, so zieh noch in der Nacht los!

28 Siehe Scholz-Cionca 1998: 231-232, 280-281. In Kinō wa kyō no monogatari sind einige der Anekdoten so derb und direkt in der Sprache, dass die chigo dort auch nichts mehr gemein haben mit den heiligen Figuren der Muromachi-Zeit. Es gibt etliche sexuell aufgeladene Witze und Doppeldeutigkeiten, die einen humorvollen Umgang mit den tatsächlichen Lebensgewohnheiten der Priester und Knaben erahnen lassen. Für eine auszugsweise Übersetzung siehe Schalow 1996: 57-65.

29 Im Nationaltheater für Nō (Kokuritsu Nōgaku-dō) gab es insgesamt 4 rekonstruierte Aufführungen, jeweils zwei von der Ōkura-Schule (1992, 2001) und zwei von der Izumi-Schule (2005, 2013). Siehe http://www2.ntj.jac.go.jp/dglib/plays/ (Suchbegriff: 老武者)

30 shidai 次第. Bezeichnung für eine kurze Ballade im kyōgen zum Auftritt einer Figur. Es stellt eine vereinfachte Imitation der shidai im Nō dar, welche dort dieselbe Funktion erfüllen. 
Sanmi: Ich lebe im Dorf Soga. ${ }^{31}$ Ferner habe ich einen noblen Tempelknaben ${ }^{32}$ bei mir und er sagt, dass er gerne die Sehenswürdigkeiten in Kamakura besichtigen möchte. Deshalb begleite ich ihn, diesen Moment reisen wir nach Kamakura.

Sanmi (michiyuki ${ }^{33}$ ): Wir verlassen das vertraute Soga. Wir verlassen das vertraute Soga. Da wir der Nase nach gehen, kommen wir zu einer nahegelegenen Unterkunft, ohne die Herberge in Fujisawa zu erreichen.

Sanmi: Ach [es ist genug], der Tag neigt sich dem Ende. Lasst uns in dieser Herberge ein Zimmer mieten.

Sanmi: Ich bitte um Einlass!

Wirt: Wer ist da?

Sanmi: Wir möchten in dieser Herberge ein Zimmer mieten.

Wirt: Ich könnte Ihnen ein ruhiges Zimmer vermieten.

Sanmi: Da wir im Geheimen reisen, vermieten Sie uns bitte ein Hinterzimmer.

Wirt: Wenn dem so ist, dann gehen Sie durch zum hinteren Bereich.

Ein Bursche: Sind alle da?

Burschen: Was ist los?

Ein Bursche: (vage in Richtung der Herberge zeigend) Es heißt, dass dort in dieser Gegend ein reizender chigo übernachtet. Da dachte ich, wir sprechen mit dem Wirt und erklären, dass wir zum chigo gehen und mit ihm Reiswein trinken wollen. Was meint ihr?

Burschen: Das ist hervorragend. Also dann, lasst uns gehen!

Ein Bursche: Wir bitten um Einlass!

31 Ein Dorf in der Provinz Sagami, heutige Präfektur Kanagawa. Es deuten noch die Großstadt Sagamihara und der Sagami-Fluss westlich von Yokohama auf die Lage der ehemaligen Provinz hin.

32 Im Text saru okata さる御方, gemeint ist der chigo.

33 michiyuki 道行. Bezeichnung für eine kurze Ballade im nō und kyōgen, bei dem die Figur den Ort und Verlauf ihrer Reise rezitiert. Ein Gang diagonal über die Bühne symbolisiert Anfang und Ende der Reise. 
Wirt: Wer ist da?

Ein Bursche: Wir sind es.

Wirt: Ach gut, ihr seid es! ${ }^{34}$

Ein Bursche: Uns wurde erzählt, dass sich ein chigo bei dir eingefunden hat. Deshalb sind wir hergekommen, um ihn um ein Trinkgelage $\mathrm{zu}$ bitten.

Wirt: Willkommen! Allerdings sagte er mir, dass er im Geheimen reist. Da er außerdem von einem sanmi begleitet wird, will ich ihn zunächst danach zu fragen. Also wartet bitte!

Wirt: Hallo. Entschuldigen Sie bitte meine bescheidene Herberge.

Sanmi: Ah, der Wirt? Kommen Sie nur herein.

Wirt: Wünschen Sie und der chigo vielleicht Gesellschaft?

Sanmi: Nein, keineswegs.

Wirt: Es scheint, dass die jungen Männer in meiner Herberge irgendwie [von dem chigo] gehört haben. Sie sagen nämlich, sie seien hergekommen, weil ein chigo hier untergebracht wurde. Sie wollen mit ihm feiern und Reiswein trinken.

Sanmi: Wie unerwartet! Aber wie ich anfangs gesagt habe, wir reisen im Geheimen. Das kommt also keineswegs in Frage.

Wirt: Dann werde ich ihnen das so ausrichten. Ich werde ihnen wie Sie gerade sagten mitteilen, dass dies ziemlich unerwartet kommt. Die Burschen werden sich wohl trotzdem uneingeladen aufdrängen. Wollen Sie deshalb nicht lieber [in diesem Zimmer] dort drüben warten?

Ein Bursche: Einverstanden. Gut, also dann lasst uns gehen.

Wirt: Als ich ihnen dies mitteilte, stimmten sie zu, dass dies wirklich unerwartet ist. Aber da Sie wohl vermitteln würden, wollen sie den Knaben unbedingt [persönlich] bitten.

34 Bei den Burschen handelt es sich um junge Männer aus der Umgebung. Der Wirt erkennt die Burschen also an der Stimme. 
Sanmi: Nein, das kommt nicht in Frage.

Wirt: Oh, sie kommen bald alle her!

Sanmi: Oh, obwohl ich von Anfang an ablehnte ... Da sie nun bald alle herkommen, lasst sie ohne etwas zu sagen direkt zu mir durch.

Ein Bursche: Ihnen mag es vielleicht äußerst unbesonnen vorkommen. Aber solche Feierlichkeiten sind doch Brauch in dieser Welt, erlauben Sie es doch bitte!

Sanmi: Nun gut, dann gebt uns den Reiswein!

(Sie veranstalten ein Trinkgelage, inzwischen möchte der chigo manju ${ }^{35}$ essen; Eine Libelle flieg ${ }^{36}$ und der sanmi schimpft „Verschwinde, verschwinde!“; Der sanmi singt und tanzt und bittet auch den chigo zum Tanz; sie tanzen wie Narren.)

Ein Greis: Es heißt, dass sich in dieser Herberge ein chigo ${ }^{37}$ einquartiert hat. Sowas ist wirklich selten! Ich will dorthin gehen und zur Freude im Alter Reiswein mit ihm trinken.

Ein Greis: Na das ist aber selten!

Ein Greis: Ob ich reinkomme? (Die Greise stehen auf der Bühne und schweigen zunächst.)

Sanmi: Ach, ist das herrlich! $!^{38}$

Greis: Bei dir hat sich ein reizender chigo ein Zimmer gemietet. Du bist ein Glückspilz! Ich bin hergekommen, weil ich zur Freude im Alter auch mit ihm Reiswein trinken möchte.

Wirt: Das ist in Ordnung. Allerdings ist gerade eine Gruppe von Burschen zugegen. Da es eine Feierlichkeit [mit allen] gibt, komm später wieder.

35 manjū 饅頭. Gedämpfte Klöße gefüllt mit süßer, roter Bohnenpaste.

36 An dieser Stelle beschreibt der chigo, dass eine Libelle umherschwirrt. Dieses zunächst seltsam anmutende Detail soll laut Nōgaku daijiten ebenso wie sein Wunsch nach Süßigkeiten die Jungenhaftigkeit des chigo unterstreichen, siehe Kobayashi et al. 2012: 954. Die Versessenheit von Knaben auf süße manjū findet sich beispielsweise auch in dem kyōgen-Stück Kanazu jizō.

37 Im Text shōjin 小人; die Bedeutung entspricht der von chigo.

38 Die Szene des Trinkgelages, das der sanmi sehr genießt, wird parallel zu dem folgenden Gespräch zwischen Wirt und Greis fortgeführt. 
Greis: Ach, welch ein Glück. Ich dachte schon fast, dass es mir nicht möglich sei ... Gehören denn die Burschen zu dieser Herberge? ${ }^{39}$

Wirt: Nein.

Greis: Was für ein großes Glück, dann werde ich auch gehen!

Wirt: Nein, wie ich gerade sagte, das geht momentan offenbar nicht. Da er außerdem hier im Geheimen ist, werde ich es dir nochmals sagen: kehr vorerst um!

Greis: Du verstehst wohl nicht recht. Wenn nur ich gehe, dann ist es doch geheim! Ich kann es nicht erwarten, zu ihm zu gehen.

Wirt: Ich habe es doch nun mehrmals abgelehnt. Du verstehst wohl nicht recht!

Greis: Das ist boshaft. Wenn du so daherredest, dann bist du in dieser Herberge nicht willkommen.

Wirt: Was Du für einen Unsinn redest! Du bist ja wohl nicht der Hausherr hier!

Greis: Da wäre ich mir nicht so sicher ... ${ }^{40}$

$-41$

Sanmi: Guten Tag. Es wäre unpassend, zu den Burschen ins Zimmer zu gehen. Kommen Sie später wieder.

Greis: Ich weiß ja nicht, wer Sie sind, aber dass Sie trotz Ihres reifen Alters so etwas [Unbedachtes] sagen? Wenn ich nicht hingehe, kann man doch gar nicht wissen, ob der chigo mich an den Festivitäten teilnehmen lässt.

Sanmi: Sie reden solch einen Unsinn! Sie sahen [zunächst] aus wie ein [reifer] Greis aus dieser Gegend, aber in Wahrheit sind Sie doch ein Quatschkopf!

Greis: Ach, Sie sind doch ein Ärgernis genau wie der Wirt!

Wirt: Er sagte euch sicherlich „Sie sind gar nicht in der Position, so etwas zu fordern!“?

39 Der Greis freut sich über die Feierlichkeit und ignoriert zunächst, dass der Wirt ihn abweist.

40 Wörtlich: Ob das so gewiss ist?

41 An dieser Stelle tritt der sanmi zum Streitgespräch zwischen Wirt und Greis und mischt sich ein. Diese Regieanweisung ist im Dramentext des Toraakibon nicht notiert, im Tenribon der Izumi-Schule wird dies aber deutlich gemacht. Siehe Ikeda/Kitahara 1974: 138. 
Greis: Es ist unglaublich!

Wirt: Sie bereuen es jetzt sicher.

Greis: Sie reden ja völligen Blödsinn! ${ }^{42}$ (Der Wirt schubst ihn beiseite)

Greis: Jetzt bin ich wütend! Euch werde ich es zeigen. Ihr werdet das bereuen! (Zwischenpause)

Wirt: Was, tatsächlich ${ }^{43}$... Es ist wirklich beschämend. Habt ihr gehört? Die Greise haben sich abgesprochen und kommen nun hier her!

Ein Bursche: Wirklich?

Wirt: Ja in der Tat.

Ein Bursche: Das ist wohl seltsam.

Wirt: Nein, es ist in der Tat so! Also bereitet euch vor!

(Die Burschen ziehen ihre kataginu ${ }^{44}$ aus und warten, jeder mit einem Stab in der Hand)

Greis (issei): Die alten Krieger mit ihren krummen Hüften, ${ }^{45}$ sie schultern wie Greise ihre verrosteten Waffen ${ }^{46}$ ! Sie stürmen heran!

Burschen: Seht unsere Vitalität, seht unsere Vitalität! Unsere Generation, die das Alte nicht kennt, ist jetzt am Zug! Wie viel Kraft euch auch gegeben sein mag, und auch wenn ihr sagt, es sei ein Leichtes für euch: Wir werden euch kräftig auslachen! (Die Burschen lachen)

Greise: Wir Greise hörten diese Begebenheiten, wir Greise hörten diese Begebenheiten: Kumasaka ${ }^{47}$ wurde mit 63 Jahren Priester, auch Saitō Sanemori ${ }^{48}$ fiel mit 60 Jahren auf

42 Der Greis versucht erbost, sich am Wirt vorbeizudrängeln

43 Der Wirt erfährt, dass die Greise angreifen wollen.

44 kataginu 肩衣. Ein ärmelloses Oberteil; typisches Kostüm im kyōgen.

45 Im Text rōmusha wa koshi ni azusa no yumi o hari; Die Assoziation von gekrümmter Haltung entsteht durch das Kopfkissenwort (makura kotoba) azusayumi 梓弓, welches eigentlich einen Bogen aus einer bestimmten Holzart (azusa 梓) bezeichnet.

46 Im Text okinasabitaru yarinaginata o, katagetsuretezo, oshiyosetaru. Okinasabitaru ist ein Schachtelwort (kakekotoba) aus okinasabu (wie alte Männer) und sabitaru yarinaginata (rostige Lanzen und Speere).

47 Kumasaka Chōhan. Bekannter Bandit gegen Ende der Heian-Zeit (794-1185). Abseits der Legenden in der Literatur gibt es keine authentischen Berichte. Siehe Ōga 1984: Bd. 6: 234.

48 Feldherr gegen Ende der Heian-Zeit. Als er die Gelegenheit hatte, Minamoto no Yoshinaka zu töten, färbte er sich Haare und Bart schwarz und starb im erbitterten Kampf auf dem Schlachtfeld. Siehe Ōga 1984: Bd. 8: 150. 
dem Schlachtfeld. Man sagt, wir alten Krieger sind [durch unser langes Leben] abgehärtet, ${ }^{49}$ und so stehen wir Euch mit ...

Ein Greis: ... 70 Jahren ...

Greise: ... oder mit ...

Ein Greis: ... 80 Jahren ...

Greise: ... in nichts nach! Wir reihen unsere Speerspitzen auf! ${ }^{50}$ (Sie rufen eitō-tō $\bar{o}^{51}$ )

Sanmi: Ich befehle euch Burschen, ich befehle euch Burschen: Es ist in der Tat recht so, ihr seid die Herren des chigo, da gibt es nichts zu zweifeln! ${ }^{52}$ Lauft und schließt die Greise in die Arme, um sie in Zaum zu halten!

Sanmi, Burschen und Wirt: Über alle Erwartung lieben sie Jünglinge, über alle Erwartung lieben sie Jünglinge. ${ }^{53}$ Wir gehen nach Hause! ${ }^{54}$ (Die Greise tragen den chigo küssend von der Bühne)

\section{Literaturverzeichnis}

Atkins, Paul (2008): “Chigo in the Medieval Japanese Imagination”. The Journal of Asian Studies 67.3: 947-970.

Childs, Margaret H (1980): “Chigo Monogatari: Love Stories or Buddhist Sermons?”. Monumenta Nipponica 35.2: 127-151.

49 Sono hoka oimusha no kūtaru tokoro ga tako ni naru そのほかおいむしやの、くうたる所が たこになる; Laut Annotation aus dem Toraakirabon eine Redewendung, deren Bedeutung bislang unbekannt ist. Laut Annotation des Tenribon wird im Kyōhō-Yasunori-bon das Wort tako mit dem ateji 胼胝 in der Bedeutung von „Hornhaut“ angegeben (ateji 当て字 sind Schriftzeichen, die nicht semantisch, sondern phonetisch verwendet werden). Zusammen mit dem Verb kū 食う in seiner Bedeutung von „etwas Unangenehmes erleiden/abbekommen“ scheint dies die sinnvollste Interpretation zu sein und wird als tentative Übersetzung übernommen. Siehe Kitagawa 1994: 349.

50 Die Annotation des Tenribon verweist auf die sexuelle Färbung dieses Ausdrucks (kissaki o soroetekakarikeri) sowie der bereits diskutierten Zeile kūtaru tokoro ga tako ni naru. Siehe Kitagawa 1994: 349.

51 Ein Kampfschrei. Die Greise greifen an und es entsteht ein wildes Gemenge auf der Bühne.

52 Der sanmi versucht, die Burschen anzufeuern, damit sie die Greise aufhalten.

53 Im Text nyakuzokuzuki 若族好き; bezieht sich hier auf die Liebe zu jungen Männern und Knaben generell.

54 In dem Getümmel scheitern die Burschen bei dem Versuch, die Greise abzuhalten. 
Dōmoto, Masaki 堂本正樹 (1976): Nanshoku engekishi 男色演劇史 (Theatergeschichte der männlichen Homoerotik). Tōkyō: Shuppansha.

Dōmoto, Masaki 堂本正樹 (1998): Zeami 世阿弥. Tōkyō: Geki Shobō.

Furukawa, Hisashi 古川久 / Kobayashi, Seki 小林責 / Ogihara, Tatsuko 荻原達子 (1976): Kyōgen jiten jikō hen 狂言辞典・事項編 (Kyōgen-Wörterbuch - Sachregister). Tōkyō: Tōkyōdō Shuppan.

Guth, Christine M.E. (1987): “The Divine Boy in Japanese Art”. Monumenta Nipponica 42.1: 1-23.

Hashimoto, Asao 橋本朝生 (2005): “Kyōgen ni miru nanshoku” 狂言に見る男色 (Männliche Homoerotik im Kyōgen-Theater). Kokubungaku kaishaku to kanshō 70.3: 132-139.

Hiramatsu, Ryūen 平松隆円 (2007). “Nihon bukkyō ni okeru sō to chigo no nanshoku”. 日本仏 教における僧と稚児の男色 (Homoerotik zwischen Priestern und Tempelknaben im japanischen Buddhismus). Bulletin of International Research Center for Japanese Studies 34: 89-130.

Ikeda, Hiroshi 池田廣司 / Kitahara Yasuo 北原保雄 (1972). Ōkura Toraakira-bon kyōgenshū no kenkyū: honmon hen jō 大蔵虎明本狂言集の研究・本文篇上 (Forschung zur kyōgenSammlung von Ōkura Toraakira, Band 1). Tōkyō: Hyōgensha.

Iwata, Jun'ichi 岩田準一 (2002). Honchō danshoku kō - danshoku bunken shoshi gappon 本朝 男色考; 男色文献書志合本 (Untersuchung zur männlichen Homoerotik in diesem Land. Gesammelte Ausgabe mit Auflistung der Literatur zur männlichen Homoerotik). Tōkyō: Hara Shobō.

Kitagawa, Tadahiko 北川忠彦 (1994): Tenribon kyōgen rikugi jōkan 天理本狂言六義上巻 (Das Tenribon kyōgen rikugi, Band 1). Tōkyō: Miyai Shoten.

Kitahara, Yasuo 北原保雄 (2000): Nihon kokugo daijiten 日本国語大辞典 (Großwörterbuch des Japanischen). Tōkyō: Shōgakukan.

Kobayashi, Seki 小林責 (2004): Kyōgenmen - kanshō to uchikata 狂言面: 鑑賞と打ち方 (Kyōgen-Masken - Ihre Wertschätzung und Herstellung). Tōkyō: Tankōsha.

Kobayashi, Seki 小林責 / Nishi, Tetsuo 西哲生 / Hata, Hisashi 羽田昶 (2012): Nōgaku daijiten 能楽大事典 (Großwörterbuch des nō- und kyōgen-Theaters). Tōkyō: Chikuma Shobō.

Kokuritsu Nōgaku-dō (Nationaltheater für nō). http://www2.ntj.jac.go.jp/dglib/plays/(30. September 2018).

Koyama, Hiroshi 小山弘志 (1990): Kyōgen kanshō annai 狂言鑑賞案内 (Anleitung zur Wertschätzung des kyōgen-Theaters). Tōkyō: Iwanami Shoten.

Koyama, Hiroshi 小山弘志 (1989): Nō kanshō annai 能鑑賞案内 (Anleitung zur Wertschätzung des nō-Theaters). Tōkyō: Iwanami Shoten.

MacDuff, William (1996). "Beautiful Boys in Nō Drama: The Idealization of Homoerotic Desire". Asian Theatre Journal 13.2: 248-258.

Ōga, Tetsuo 相賀, 徹夫 (1984): Nihon dai hyakka zensho 日本大百科全書 (Encyclopedia Nipponica). Tōkyō: Kogakukan.

Schalow, Paul Gordon (1996): “Today's Tales of Yesterday”. In: Partings at Dawn - An Anthology of Japanese Gay Literature. Hrsg. von Stephen Miller. San Fransisco: Gay Sunshine Press, 57-65.

Scholz-Cionca, Stanca (1998): Entstehung und Morphologie des klassischen Kōgen im 17. Jahrhundert- Vom mittelalterlichen Theater der Außenseiter zum Kammerspiel des Shogunats. München: ludicium. 